\title{
RESEARCH ON ACCURACY EVALUATION METHOD BASED ON THE RESULT OF IMAGE CORRECTION AT 10000-SCENES CLASS
}

\author{
LI Miao ${ }^{1}$, CHEN HaiPeng ${ }^{1}$, QIU Bo ${ }^{1}$, XIE WenJun ${ }^{1}$, CHEN YuHeng ${ }^{1}$
}

${ }^{1}$ National Quality Inspection and Testing Center for Surveying and Mapping Products, 9105513@qq.com

\section{Commission VI, WG VI/4}

KEY WORDS: Orthorectification, Quality control, Accuracy, 10000-Scenes class

\section{ABSTRACT:}

In the project of National Fundamental Geographic Information Database Updating, the high-qualified orthorectification to a bulk of remote sensing images are the foundations that guarantee the reliable geographic information for national economic construction and social developments. Therefore, quality control for orthorectification, as a crucial step during remote sensing, is of great significance. Basically, the image orthorectification have been achieved by automatic matching of millions of frame-referenced images. In this paper, a make-to-measure method is devised with improvement in the way of sampling and plane precision evaluation. The improved method is verified through nine 1:1million scenes across the nation. Stratified sampling is deployed according to the topographical features and locations geo-information is collected referring to the digital orthorectification model (DOM) results of geographical conditions Monitoring in 2017. The results show that the root mean square errors (RMSEs) calculated by improved methods described in this paper are highly consistent with the RMSEs that are calculated by automatic image matching, which means the improved method can adequately evaluate the nationwide accuracy of image correction and enable to provide reference and instruction for the large-scale, 10000-scenes class quality assurance of the image correction.

\section{INTRODUCTION}

National basic geographic information is the focus of national spatial data infrastructure construction, The National Basic Geographic Information Database is updated annually for the entire country, we will collect and obtain domestic satellite remote sensing images such as ZY-3 covering the whole country, complete orthorectification of panchromaticchromatic images and multi-spectral images in the form of scenery, forming the results of national satellite image orthorectification processing.

As the basic image data of the 1:5 million terrain data update in the country, the quality level of image orthorectification processing results directly affects the terrain data update work and the final result quality $^{[1]}$. Carrying out quality inspection of results is an important means to discover the quality of results and to ensure the quality of results. Due to the particularity of image ortho-correction processing results, targeted inspection techniques and methods are required to highlight the pertinence and effectiveness of quality inspection while ensuring comprehensive coverage of quality requirements. Based on the actual inspection work, considering the operability and labor cost in scale production, this paper proposes a quality inspection and evaluation method based on the large-scale ortho-corrected image of 10000-Scenes class. Since the focus is on the accuracy of the mapping results when using the satellite as the basic mapping update, this paper focuses on the accuracy evaluation method of image correction results.

\section{SATELLITE IMAGE POSITIONING ACCURACY}

When satellite images are used as the basic mapping update, it is necessary to further understand the relevant performance indicators of satellite images. In addition to considering the accuracy of the mapping results, it is also necessary to consider various factors such as operability and labor cost in scale production. The ground positioning accuracy of satellite images refer to the accuracy index that can be achieved by determining the position of the ground point based on satellite images. It is related to the spatial resolution of the image, the geometric distortion of the image, and the image quality ${ }^{[2]}$.

\subsection{Image Spatial Resolution}

Spatial resolution, also known as ground resolution, is 
an important indicator of satellite image accuracy. The spatial resolution determines the size of the smallest cell unit, which is the size of the pixel. The higher the resolution, the more pixels are contained in the unit area of the surface, and the richer the information, the stronger the ability to distinguish the features. The effects of spatial resolution of satellite images on the update of basic surveying data are:

(1) The precise positioning ability of the control point with the same name image;

(2) The ability to distinguish small features can meet the corresponding scale topographic map update requirements.

The ground resolution of the satellite image used for updating 50,000 terrain data is shown in Table 1 .

\begin{tabular}{|l|c|c|}
\hline $\begin{array}{c}\text { Satellite } \\
\text { Source }\end{array}$ & $\begin{array}{c}\text { Resolution } \\
\text { (panchromatic) }\end{array}$ & Resolution(mul) \\
\hline ZY3 & 2.1 & 5.8 \\
\hline GF1 & 2 & 8 \\
\hline GF2 & 0.8 & 3.2 \\
\hline TH1 & 2 & 10 \\
\hline
\end{tabular}

Table 1. Satellite images ground resolution (meter)

\subsection{Image Geometric Correction Accuracy}

Geometric correction is to eliminate the geometric distortion of the image, that is, to transform the image coordinates into map coordinates. The geometric correction accuracy is an indicator reflecting the geometry of the ground image after satellite image correction and its spatial positioning capability. The main factors affecting geometric accuracy are image quality/quantity of control points and DEM accuracy. Orthophoto correction of image accuracy needs to meet the requirements of 1:50,000 scale mapping. The accuracy of the plane position of 1:50,000 scale is: flat land and hills are 25 meters, mountain and Alpine land are 37.5 meters. 1:50,000 orthophotos in the position of the error shall not be greater than the provisions of Table 2 .

\begin{tabular}{|c|c|}
\hline Terrain Type & $\begin{array}{c}\text { Plane position } \\
\text { RMSEs }\end{array}$ \\
\hline flat land /hills & 25 \\
\hline mountain / Alpine land & 37.5 \\
\hline
\end{tabular}

Table 2. Plane accuracy

For a large area of a single feature area, such as a water body, the error can be appropriately relaxed, but the maximum should not be greater than 1.5 times of the above table; for areas with special image such as poor image texture and forest, high-rise building coverage area, the error in the plane position can be relaxed. However, the maximum shall not be greater than 1.5 times of the above table, and the error of 2 times shall be the maximum error.

When the same track images or the different track images are edged, the indirect edge tolerance of the scene can be performed according to Table 3 .

\begin{tabular}{|c|c|}
\hline Terrain Type & Edge error \\
\hline flat land /hills & 25 \\
\hline $\begin{array}{c}\text { mountain / Alpine } \\
\text { land }\end{array}$ & 37.5 \\
\hline
\end{tabular}

Table 3. Edge error

\subsection{Image Texture Quality}

The imaging quality of satellite images are mainly affected by factors such as ground illumination, cloud fog and flight attitude during photography. It can be evaluated and checked from the following aspects:

(1)The image signal-to-noise ratio is small, the linearization in the dynamic range is better, the gray level is more, the image level is richer, and the resolution of the target in the shadow is improved.

(2) The image tilt angle should be small to reduce the deformation and blur of the back slope image; Cloud or fog coverage is generally less than $10 \%$, and more than $10 \%$ need to try to supplement images.

\section{STECHNICAL ROUTE DESIGN}

The satellite image ortho-correction treatment results cover the country's 1:50,000 topographic map area, including Hong Kong, Macao and Taiwan, with an area of about 9.6 million square kilometers and a data volume of nearly 20,000 . In the face of such large-scale and large-volume data, in addition to ensuring the quality evaluation indicators, we must also consider the operability and labor costs in this normal-scale production. In the exploration process of the inspection method, the technical route adopted by the production is fully analyzed, and the geometrical precision inspection technical method design and research are carried out by combining the production technology route and the project quality requirements. 


\subsection{Production Technology Route Analysis}

In the production, the precision correction and analysis of the regional network adjustment orthorectification results based on high-precision control points in recent years are carried out. The RMSEs in the image plane position is less than $5 \mathrm{~m}$, and it has reliable geographical reference accuracy. The previous results are used as a reference for correction image in the 1:50,000 terrain data update, the reference images are mosaiced in a million standard frame, and a total of 63 million frames are formed nationwide. The panchromatic image orthorectification is mainly based on the image of the million-frame reference image. The matching is corrected, and then the control point matching, editing and orthorectification processing corresponding to the multi-spectral image are completed.

\subsection{Overall Technical Route}

In the process of plane position accuracy detection, comprehensively consider the use of higher-precision geographical images, production-corrected reference images as reference data for accuracy evaluation, supplemented by panchromatic image and multi-spectral image nesting inspection. Inspection and evaluation, the process is shown in Figure 1.

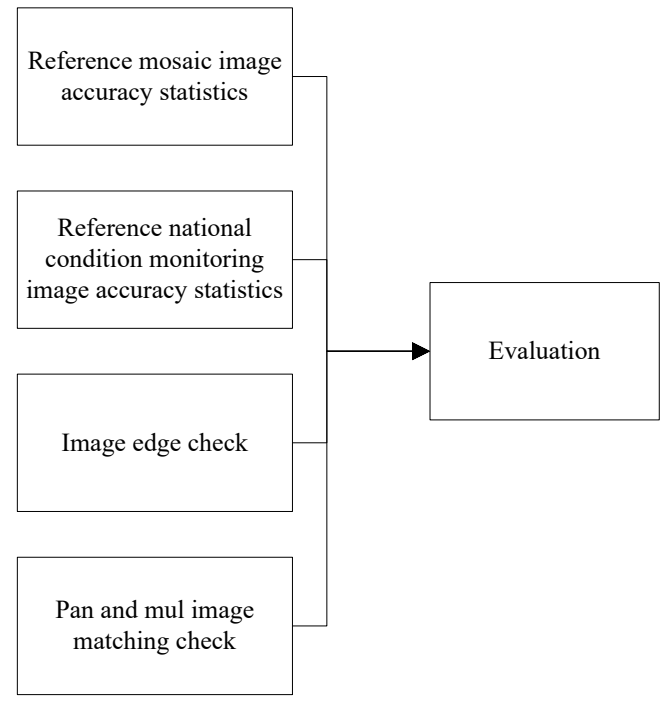

Figure 1. Plane position accuracy check method

\section{EXPERIMENTAL METHODS AND}

RESULTS ANALYSIS

\subsection{Sampling Method}

In the 1:50,000 terrain data update, the reference image is inlaid in millions of standard frames, and the positional accuracy is referenced to the production technology route for hierarchical sampling.According to the topographical features, 9 million maps are evenly extracted across the country according to a certain proportion, so that the sample map covers different regions of china, including plains, hills, plateaus and other terrains, on the basis of the one-stage sampling, the satellite type and the result batch are taken into two-stage proportional sampling.

\subsection{Plane Position Accuracy Check Method}

In the plane position accuracy detection process, through the use of internal inspection and reference data comparison method, the plane position accuracy is comprehensively analyzed in various ways, which has become a complete and scientific precision verification process. Comprehensively use higher precision image results, reference images, etc. as reference data for accuracy evaluation ${ }^{[3]}$,

supplemented by panchromatic images and multi-spectral images for nesting inspection.

(1)The production images used in the production are corrected as reference data for accuracy detection, and the panchromatic correction results and reference images are superimposed and displayed, and 300 points of the same name are automatically selected by the software, and the computer automatically calculates the RMSEs in the single-view image.

(2)The third-party results are used for verification, and the high-precision images in 2017 are used as reference images to accurately detect panchromatic images. 5 scene images are extracted from each million frames, and collect 20 points of the same name for each image, a total of about 900 detection points are collected, and the RMSEs of each scene image is counted in units of 1:100 million.

(3)In order to ensure the fusion effect, set and multi-spectral and full-color images, measure whether the same-named point is accurate to the sub-pixel precision.

\subsection{Edge Accuracy Evaluation Method}

The result image has a overlapping edge and the entire overlap is not completely connected,partially incomplete connected, and the edge is not connected to the edge. Therefore, the RMSEs in the edge of GB/T18316-2008 ${ }^{[4]}$ cannot be directly used for quality evaluation,but refer to GB/T24356-2009 [5], and the quality assessment is carried out by the wrong-leakage 
deduction method. That is, the four types of faults A, $\mathrm{B}, \mathrm{C}$, and $\mathrm{D}$ are determined according to the specific conditions of the joint edge problem, as shown in Table 4

\begin{tabular}{|c|c|c|c|c|}
\hline $\begin{array}{l}\text { Check } \\
\text { item }\end{array}$ & $\mathbf{A}$ & B & C & D \\
\hline $\begin{array}{l}\text { Image } \\
\text { edge }\end{array}$ & $\begin{array}{l}\text { 1.The } \\
\text { overall } \\
\text { edge is } \\
\text { more than } \\
2 \text { pixels } \\
2 . \text { Other } \\
\text { serious } \\
\text { mistakes }\end{array}$ & $\begin{array}{l}\text { 1.The whole is } \\
\text { not connected, } \\
\text { and the edge } \\
\text { error does not } \\
\text { exceed } \\
\text { pixels.; } \\
2 . \text { Partially not } \\
\text { connected, } \\
\text { and the edge } \\
\text { error exceeds } \\
2 \text { pixels; } \\
\text { 3.Other } \\
\text { heavier errors }\end{array}$ & $\begin{array}{l}\text { 1.Partially } \\
\text { not } \\
\text { connected, } \\
\text { and the edge } \\
\text { error does } \\
\text { not exceed } 2 \\
\text { pixels; } \\
\text { 2.Other } \\
\text { general } \\
\text { errors }\end{array}$ & $\begin{array}{l}\text { Other } \\
\text { minor } \\
\text { errors }\end{array}$ \\
\hline
\end{tabular}

Table 4. Edge error classification table

\subsection{Result Analysis}

The panchromatic image of the single-view is calculated in units of millions of frames using the corrected reference image for production as the reference data, and the mean value is used as the medium error value of the million-frame in the mode. The national monitoring image results are used as reference images to analyze and compare the errors in the unit of millions of frames. The results of the errors are shown in Table5 and Figure 2.

\begin{tabular}{|c|c|c|}
\hline $\begin{array}{c}\text { Map } \\
\text { number }\end{array}$ & $\begin{array}{c}\text { mosaic image } \\
\text { RMSEs (meter) }\end{array}$ & $\begin{array}{c}\text { monitoring image } \\
\text { RMSEs (meter) }\end{array}$ \\
\hline H46 & 2.93 & 8.08 \\
\hline H50 & 2.82 & 4.18 \\
\hline I48 & 4.05 & 5.18 \\
\hline I49 & 3.50 & 4.16 \\
\hline J47 & 2.73 & 2.16 \\
\hline K45 & 2.30 & 2.37 \\
\hline K46 & 5.88 & 1.17 \\
\hline K50 & 3.9 & 2.77 \\
\hline L52 & 3.73 & 2.29 \\
\hline
\end{tabular}

Table 5. Panchromatic image position accuracy

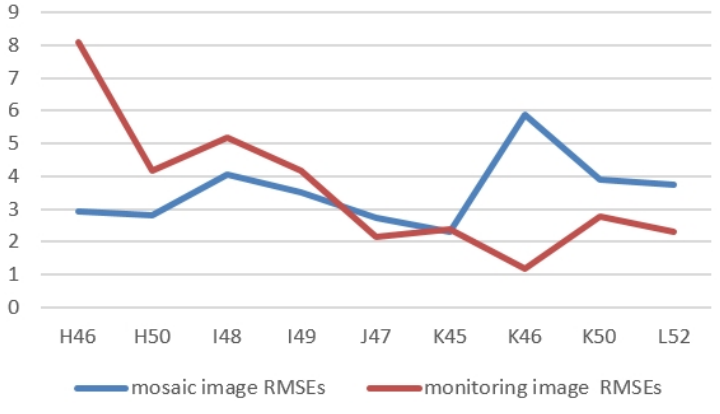

Figure 2. Image accuracy analysis result

Through the analysis of the accuracy test results, the main conclusions are drawn:

Whether it is the use of higher-precision geography orthophoto results, or the use of corrected reference images as reference data for accuracy evaluation, the accuracy level obtained by the two methods is basically the same, and the RMSEs is less than $1 / 3$ of the allowable error, and it can capable of objectively responding to the accuracy and quality of satellite image orthorectification processing results in 1:50,000 terrain data update.

Some of the millions of frames such as H46 statistical results refer to the RMSEs of the reference million frame mosaic image is smaller than the reference error of the reference geographical situation monitoring image, there are three reasons for analysis: First, the feature points of the regions where the image samples are taken are not obvious, which brings difficulties to manually collecting the same-named points, and there is a problem that the error of the same name points exceeds the limit. The second is to use software to automatically match the same-named point of the ortho-corrected image and the million-frame reference image, and gradually eliminate the gross difference in the matching process, and retain the effective same-named point to ensure high matching accuracy. Third, due to the special requirements of the national conditions monitoring image results, in the areas where the two images are not changed and the special difficulties are difficult, the image position accuracy is allowed to be appropriately relaxed. These reasons have led to the possibility that the geographical monitoring results of this region have less than the accuracy of the reference image of millions of frames.

\section{CONCLUSION}

This paper analyzes the satellite image ground positioning accuracy evaluation index when updating 
the basic surveying and mapping data, and proposes a sampling, plane position precision quality inspection and evaluation method for the large-scale satellite image orthorectification processing results. The second is to use software to automatically match the same-named point of the ortho-corrected image and the million-frame reference image, and gradually eliminate the gross difference in the matching process, and retain the effective same-named point to ensure high matching accuracy.

(1)Combined with the production route, a sampling method was designed for targeted improvement, and two-stage sampling was carried out based on topographical features, satellite types and outcome batches.

(2)Through the reference to the geographical situation monitoring DOM results, production correction reference images, etc. as reference data for accuracy evaluation, supplemented by panchromatic image and multi-spectral image nesting inspection for comprehensive inspection and evaluation. The sampling method is applicable to projects with large scope and large data. Through the design of strict position accuracy verification method, the accuracy evaluation problem of satellite image orthorectification processing results when the field control points are not easily obtained is effectively solved.

By using the technical methods of this paper to carry out the actual work, the results of the orthorectification correction processing in the 1:50,000 terrain data update are tested and evaluated, which proves that the route is feasible and the quality evaluation results are objective and reliable, and can correct the large-scale and 10000-Scenes class images.
The quality inspection and evaluation of the treatment results provide reference and method guidance.

\section{REFERENCES}

[1].CHENG Tao,GUO YaLin,ZHOU Xu.A quality inspection method for mass digital orthphoto maps [J].Journal of HeFei University of Technology.2013, $36(7): 816-817$.

[2].WANG WenWen,ZHANG YongHong.Geometric quality evaluation of CBERS202B[J].Bulletin of Surveying and Mapping.2009,11:11-13.

[3].CHENG PengFei,ZHAO YouSong, YIN Su.Key technology of quality inspection in the production of coastal island(rock) DEM [J]. Bulletin of Surveying and Mapping.2015 (9):48-50.

[4]. General Administration of Quality Supervision, Inspection and Quarantine of the People's Republic of China, Standardization Administration of the People's Republic of China. GB/ T 18316-2008, Specfications for inspection and acceptance of quality of digital surveying and mapping achievements[S], BeiJing: Standards Press of China, 2008.

[5]. General Administration of Quality Supervision, Inspection and Quarantine of the People's Republic of China, Standardization Administration of the People's Republic of China. GB/ T 24356-2009, Specifications for quality inspection and acceptance of surveying and mapping products[S], BeiJing: Standards Press of China, 2009. 